\title{
Online Multi-Agent Pathfinding
}

\author{
Jiří Švancara, ${ }^{1}$ Marek Vlk,,${ }^{1,2}$ Roni Stern, ${ }^{3}$ Dor Atzmon, ${ }^{3}$ Roman Barták ${ }^{1}$ \\ ${ }^{1}$ Faculty of Mathematics and Physics, Charles University, Prague, The Czech Republic \\ ${ }^{2}$ Czech Institute of Informatics, Robotics and Cybernetics, Czech Technical University in Prague, The Czech Republic \\ ${ }^{3}$ Information Systems Engineering, Ben Gurion University, Be'er Sheba, Israel \\ \{svancara, vlk, bartak\}@ktiml.mff.cuni.cz, \{sternron, dorat\}@ post.bgu.ac.il
}

\begin{abstract}
Multi-agent pathfinding (MAPF) is the problem of moving a group of agents to a set of target destinations while avoiding collisions. In this work, we study the online version of MAPF where new agents appear over time. Several variants of online MAPF are defined and analyzed theoretically, showing that it is not possible to create an optimal online MAPF solver. Nevertheless, we propose effective online MAPF algorithms that balance solution quality, runtime, and the number of plan changes an agent makes during execution.
\end{abstract}

\section{Introduction}

Multi-agent pathfinding (MAPF) is the problem of moving a group of agents to a set of target destinations while avoiding collisions (Silver 2005). MAPF has applications in robotics, avionics, digital entertainment, and more, and it has attracted significant research focus from various research communities in recent years (Pallottino et al. 2007; Erdem et al. 2013; Surynek et al. 2016; Standley 2010; Sharon et al. 2015).

Most work on MAPF has focused on finding a plan for all the agents before the agents start to move (Felner et al. 2017; Surynek et al. 2016; Yu and LaValle 2012). The agents are then expected to follow that plan until eventually each agent reaches its designated goal. We refer to this a priori planning problem as offline MAPF. In this work, we consider an online version of MAPF, where new agents may appear while the other agents are following a previously generated plan.

Online MAPF is a natural generalization of offline MAPF with applications in controlling fleets of vehicles or teams of robots. For example, consider the autonomous intersection management (AIM) project by Dresner et al. (2008). An autonomous agent controls an intersection, and driver agents that wish to pass through the intersection must follow the intersection manager's directions. Once an agent passes the intersection, it leaves the system, while new agents may enter the intersection. The intersection manager, thus, is actually solving an online MAPF problem. The general approach they used is a first-come first-serve approach, which is clearly not optimal.

Copyright (C) 2019, Association for the Advancement of Artificial Intelligence (www.aaai.org). All rights reserved.
Online MAPF is related to the lifelong MAPF setting studied by Ma et al. (2017). In lifelong MAPF the set of agents do not change, but over time the problem solver receives a sequence of navigation tasks that they need to perform (e.g., get from one location to another). Thus, task assignment plays an important role in lifelong MAPF. In our setting, we focus on the intersection problem, where new agents can appear, and there is no need for task assignment as every new agent is associated with a specific start and goal.

The first contribution of this work is in a formal definition and analysis of the online MAPF problem. We discuss several possible objective functions and show equivalences between them. Several variants of online MAPF are defined and analyzed, showing which variants allow creating complete and optimal solvers and which do not.

Our second contribution is several practical solvers for online MAPF and the discussion on their properties. In particular, we propose a SAT-based solver using the Picat language and a solver based on the Conflict-Based Search (CBS) (Sharon et al. 2015). To minimize the number of times agents change their routes, we introduce the Online Independence Detection algorithm that biases the resulting plan to cause fewer disruptions to the currently executed plans, while still guaranteeing a high quality solution.

The third contribution of this work is an online MAPF algorithm that allows trading off solution quality for the amount of disruptions to other agents. We evaluate all the proposed algorithms on grids of different size and with a varying number of agents and obstacles. The results show the trade-offs for the different solvers and problem parameters.

\section{Background: Offline MAPF}

An offline MAPF problem accepts as an input a graph $G=$ $(V, E)$ and a set of agents $a_{1}, \ldots a_{n}$, such that each agent $a_{i}$ is associated with an initial location $s_{i}$ and goal location $g_{i}$. Time is discretized, and in every time step each agent can either move from its location to an adjacent location, or wait in its current location. A solution to offline MAPF is a plan $\pi$ that consists of a sequence of wait/move actions for each agent. Let $\pi_{i}[j]$ denote the location agent $i$ will reach after performing the first $j$ actions in the plan $\pi$, ignoring all other agents. The main constraint in offline MAPF is that 
the agents must not collide, i.e., occupy the same vertex or edge at the same time. A plan $\pi$ has a collision if there is a time step $j$ in which there are two agents $i_{1}$ and $i_{2}$ that are planned to occupy the same location or traverse the same edge. Formally, agent $i_{1}$ and $i_{2}$ collide at time $j$ in plan $\pi$ if

$$
\begin{aligned}
& \left(\pi_{i_{1}}[j]=\pi_{i_{2}}[j]\right) \vee \\
& \quad\left(\left(\pi_{i_{1}}[j]=\pi_{i_{2}}[j+1]\right) \wedge\left(\pi_{i_{1}}[j+1]=\pi_{i_{2}}[j]\right)\right)
\end{aligned}
$$

A solution to an offline MAPF is such a plan $\pi$ that has no collisions. We refer to such a plan as a valid plan, and denote by $\left|\pi_{i}\right|$ the number of actions in $\pi_{i}$. A given offline MAPF problem may have many solutions, and not all are equally preferable. It is common to associate a MAPF solution $\pi$ with a cost according to some objective function, so that solutions with minimal cost are preferred. The two most common objective functions are makespan and sum of costs (SOC). The former is the time required to have all agent reach their goals (Makespan $(\pi)=\max _{i=1}^{n}\left|\pi_{i}\right|$ ), and the latter is the sum of times required for each agent to reach its goal (the sum of $\operatorname{costs}(\pi)=\sum_{i=1}^{n}\left|\pi_{i}\right|$ ).

\section{Problem Definition}

An online MAPF problem starts with an offline MAPF problem, which an online MAPF solver must first solve. Then, while the agents execute the generated plan, a sequence of new agents appear on the map. The $i^{t h}$ new agent is defined by the triplet $\left\langle t_{i}, s_{i}, g_{i}\right\rangle$, where $t_{i}$ is the time step in which the agent appears, $s_{i}$ is its initial location, and $g_{i}$ is its goal location. Importantly, this sequence of new agents is given to the problem solver online, such that only at time $t_{i}$ it is revealed that a new agent wishes to get from $s_{i}$ to $g_{i}$. Thus, this part of the problem input - the new agents - is referred to as the online input of an online MAPF problem, while the graph and the initial set of agents is referred to as the offline input.

A solution to an online MAPF problem is a sequence of valid plans $\Pi=\left\langle\pi^{0}, \pi^{1}, \ldots, \pi^{m}\right\rangle$, where $m$ is the number of times a new agent appears, $\pi^{0}$ is the plan created for the offline input, and $\pi^{i}$ for $i>0$ is the plan created when the $i^{\text {th }}$ new agent appears at time $t_{i}$. A partial plan $\pi^{i}[x: y]$ is the part of the plan $\pi^{i}$ that is planned for time steps $x, x+1, \ldots, y$. We assume that the agents follow the most recently generated plan, i.e., after the $i^{t h}$ agent appears, all agents follow plan $\pi^{i}$ until the $(i+1)^{t h}$ appears, after which the agents follow plan $\pi^{i+1}$. Thus, the plan the agents end up executing, denoted $E x[\Pi]$, can be written as $\operatorname{Ex}[\Pi]=\pi^{0}\left[0: t_{1}\right] \circ \pi^{1}\left[t_{1}+1: t_{2}\right] \circ \ldots \circ \pi^{m}\left[t_{m}+1: \infty\right]$, where $\circ$ represents concatenation of partial plans. We refer to $E x[\Pi]$ as the execution of $\Pi$. A solution to an online MAPF problem is a sequence of plans $\Pi$ such that $E x[\Pi]$ forms a valid plan (i.e., without collisions).

\section{Online MAPF Variants}

One can imagine many variants of online MAPF, in particular with respect to (1) what happens when an agent reaches the goal and (2) what happens when a new agent appears in its initial location.

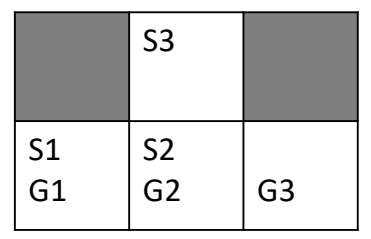

Figure 1: An instance that is solvable by the offline optimal solver, but cannot be solved by any online MAPF solver.

Consider first the question of what happens when an agent reaches its goal. One option is that when an agent reaches its goal it stays there. This results in a setting similar to the mentioned above lifelong MAPF (Ma et al. 2017). A different option is that an agent disappears when reaching its goal. Such an assumption makes sense when the goal is associated with some location that the agent can actually enter and stay there without interfering others, e.g., a private parking space.

Orthogonal to the decision of what happens to an agent when it reaches the goal is the decision of what happens when a new agent appears. One option is to assume that a new agent immediately appears in its initial location. This can cause unavoidable collisions, as another agent may already be in that location when the new agent appears. A different assumption regarding new agents is that when a new agent appears it needs to perform a move action in order to enter its start location, and it can also wait as long as it wishes before doing so. This assumption also corresponds to a private parking space scenario, where the agent can wait in it, e.g., if it sees that its initial location in the graph is already occupied. We refer to this private place as the agent's garage.

Consider the assumption that an agent disappears at its goal and the assumption that the agent appears in its garage. These correspond to a scenario where there is a private part of the world that is not managed in a centralized way, and the agents start from and wish to go to such private locations. To do so, the agents must pass through a public area that is controlled by some autonomous agent, e.g., an autonomous driving scenario where only the traffic in the city centers is fully automatized (Dresner and Stone 2008).

Next, we analyze the online MAPF problem under each of these four combinations of assumptions - staying at the goal or disappearing and appearing in the grid or in the garage.

\section{Problem Analysis}

A common way to analyze online problems and algorithms is to consider the behavior of an offline optimal solver for this problem. An offline optimal solver is one that accepts all the inputs to the online problem upfront. In our case, an offline optimal solver for online MAPF knows in advance when new agents will arrive and what will be their start and goal locations. One can easily modify any offline MAPF solver to serve as an offline optimal solver. Clearly, an offline optimal solver cannot be used in practice, but it is useful for analysis as clearly no online MAPF solver can do any better than the offline optimal solver. 


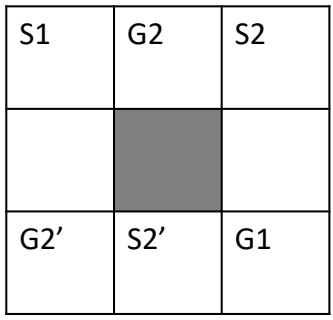

Figure 2: An instance in which no online MAPF solver can return the optimal solution.

\begin{tabular}{l|l|l} 
Appears & Stay at goal & Disappear \\
\hline In grid & $\begin{array}{l}\text { Collisions } \\
\text { \& Incomplete } \\
\text { (Obs. 1) }\end{array}$ & Collisions \\
\hline In garage & $\begin{array}{l}\text { Incomplete } \\
\text { (Obs. 1) }\end{array}$ & Complete (Prop. 2)
\end{tabular}

Table 1: Summary of theoretical results for online MAPF

Observation 1. If agents do not disappear when they reach their goals, then there are problem instances that are solvable by an offline optimal solver but cannot be solved by any complete online MAPF solver.

Proof. Consider an online MAPF problem with 3 agents, situated in the grid given in Figure 1. $S 1, S 2$, and $S 3$ are the start locations of agents 1,2, and 3, respectively. Similarly, $G 1, G 2$, and $G 3$ are the agents' goal locations. Now, assume that agent 1 appears first, then agent 2 , and finally agent 3 . An offline optimal solver will have agent 2 wait in its private space until agent 3 appears and reaches its goal. By contrast, any complete online MAPF solver will have to eventually decide to move agent 2 to its goal. We can construct an online MAPF problem that will have agent 3 appear only after this has occurred. When this occurs, the problem becomes unsolvable, as agent 3 can only reach its goal if agent 2 has not entered its starting location.

Proposition 2. An online MAPF problem where agents disappear at the goal and where new agents may wait before entering their initial location is solvable iff the offline part of the problem is solvable, assuming there exists a path for each agent from its initial location to its goal location.

Proof. If the offline part is solvable, we can then just let the new agents wait in their garages until all of the other agents disappear. The problem is then reduced to single agent shortest path. The other implication is trivial.

Since assuming that an agent appears immediately on the grid may cause unavoidable collisions, and assuming that the agent stays at the goal may lead to instances that no online solver can solve (Observation 1), we decided to focus the rest of this paper on online MAPF where the new agents appear in their garage and agents that reach their goal disappear. Note that waiting in a garage to enter the graph counts as an action in the plan. Table 1 summarizes our observations for the four combination of assumptions.

\section{Objective Functions}

Now we ask the question of how to evaluate a solution $\Pi$ for an online MAPF problem. Porting the sum of costs measure from offline MAPF to online MAPF is straightforward: the sum of costs of an online MAPF solution $\Pi=$ $\left\langle\pi^{0}, \pi^{1}, \ldots, \pi^{m}\right\rangle$ is defined as the sum of costs of the executed plan $E x[\Pi]$, i.e., $f_{1}(\Pi)=\sum_{i \in A}\left|E x[\Pi]_{i}\right|$, where $\left|E x[\Pi]_{i}\right|$ is the number of steps the $i^{t h}$ agents took until it reached its goal.

Porting the makespan measure, however, is problematic, since makespan is defined as the arrival time of the last agent to its goal position. Due to the online nature of online MAPF, agents may keep emerging infinitely, resulting in Makespan $=\infty$, which is clearly undesirable. We now propose several possible objective functions intended to fill this gap. Let $A$ be the set of agents, NotAtGoal( $t)$ be the number of agents that are not at their goals at time step $t$ yet, and $o_{i}$ be the length of the optimal (shortest) path for the $i^{\text {th }}$ agent when no other agent is present. For a plan $\Pi$ and its execution $E x[\Pi],\left|E x[\pi]_{i}\right|$ is the number of steps the $i^{t h}$ agent took until it reached its goal.

\section{- Sum of agents not at goal over time.}

$$
f_{2}(\Pi)=\sum_{t=1}^{\infty} \operatorname{NotAtGoal}(t)
$$

- Sum of times over individual cost.

$$
f_{3}(\Pi)=\sum_{i \in A}\left|E x[\Pi]_{i}\right|-o_{i}
$$

Definition 3 (Objective Function Equivalence). A pair of objective functions $f_{x}$ and $f_{y}$ are equivalent if for every online MAPF problem and every pair of solutions $\Pi$ and $\Pi^{\prime}$ for that problem, we have

$$
f_{x}(\Pi) \leq f_{x}\left(\Pi^{\prime}\right) \Leftrightarrow f_{y}(\Pi) \leq f_{y}\left(\Pi^{\prime}\right)
$$

Proposition 4. All the objective functions listed above are equivalent to the sum of costs.

Proof. " $f_{1} \Leftrightarrow f_{2}$ " Let $\chi_{i}^{t}$ be 1 if the $i^{\text {th }}$ agent is not yet at its goal location at time $t$, otherwise 0 . Then $f_{1}(\Pi)=$ $\sum_{i \in A} \sum_{t=1}^{\infty} \chi_{i}^{t}$ and $f_{2}(\Pi)=\sum_{t=1}^{\infty} \sum_{i \in A} \chi_{i}^{t}$. Hence, by swapping the sums, $f_{1}(\Pi)=f_{2}(\Pi)$ for arbitrary $\Pi$.

" $f_{1} \Leftrightarrow f_{3}$ " Since $f_{1}(\Pi)=\sum_{i \in A}\left|E x[\Pi]_{i}\right|=$ $\sum_{i \in A}\left|E x[\Pi]_{i}\right|-o_{i}+\sum_{i \in A} o_{i}=f_{3}(\Pi)+\sum_{i \in A} o_{i}$, and because $\sum_{i \in A} O_{i}$ is a constant depending just on the problem and not on the solution, it holds that $f_{1}\left(\Pi_{x}\right) \leq f_{1}\left(\Pi_{y}\right) \Leftrightarrow$ $f_{3}\left(\Pi_{x}\right) \leq f_{3}\left(\Pi_{y}\right)$ for arbitrary plans $\Pi_{x}, \Pi_{y}$.

Thus, we only discuss the sum of costs objective function $\left(f_{1}\right)$ for online MAPF, and refer to a solution as optimal if it minimizes the sum of costs.

Observation 5. There is no complete online MAPF solver that can guarantee to return a solution with a cost equal to the offline optimal MAPF solver. 
Proof. Consider an online MAPF problem with 2 agents, situated in the grid given in Figure 2. $S 1$ and $G 1$ are the start and goal of agent 1 , which is the first agent to appear. The agent has two shortest paths to reach its goal: going right and then down, or going down and then right. After the agent chooses one of these options, the second agent appears, either in S2 or in S2'. An offline optimal solver will know upfront where the second agent will appear and choose appropriately if the first agent will go down and right (avoiding S2) or right and down (avoiding S2'). An online MAPF solver cannot know this in advance and is thus bound to yield a suboptimal solution.

\section{Online MAPF Algorithms}

While Observation 5 states that guaranteeing an optimal solution is not possible with a complete solver, there is still a need to solve online MAPF problems in some principled way. In this section, we propose several online MAPF algorithms and discuss their properties.

The offline part of the problem, i.e., planning for the initial set of agents, can be done by a different algorithm than the one used for the online part. We focus our discussion on the online part and assume that all algorithms we propose start with an optimal solution to the initial offline problem. Hence, in what follows we only describe only the replan function, which is called when new agents appears. The input to such a replan functions is always the set of current agents $A$, the set of new agents $A^{+}$, and the ongoing plan $\pi_{A}$, which is the plan the agents in $A$ are currently following. Note that more than one new agent may appear at the same time, and thus $A^{+}$may contain multiple agents.

\section{Replan Single and Replan Single Grouped}

The first two algorithms we describe serve as a baseline. The Replan Single (RS) algorithm searches for an optimal path for each new agent, one at a time, while avoiding all other (already planned) agents. The Replan Single Grouped (RSG) algorithm searches for optimal paths for all new agents at once.

To formally describe RS and RSG, we introduce the following helping notation. Let $\psi_{A}^{\pi_{B}}$ be an optimal plan for a group of agents $A$ while avoiding some plan $\pi_{B}$ for a group of agents $B$. This assumes that the groups $A$ and $B$ are disjoint. In particular, $\psi_{A}^{\emptyset}$ means an optimal plan for the agents in group $A$ without considering any agent that is not in $A$. Algorithms 1 and 2 list the pseudo codes for RS and RSG using our helping notation $\psi_{A}$ appropriately. Note that when only one new agent appears, RS and RSG behave in the same way.

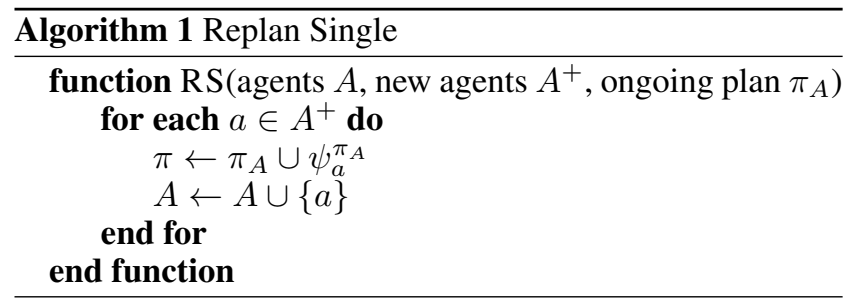

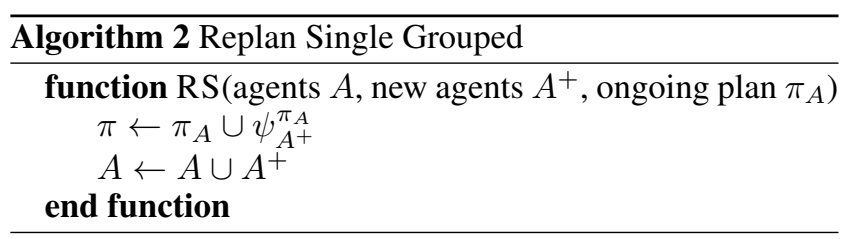

Analysis. RS can be solved in polynomial time, since it runs one single-agent path finding search. Therefore, in our implementation of the rest of the algorithms described in this work, we first run RS to obtain a baseline solution, and then try to improve on it in the rest of the runtime.

RSG, on the other hand, may require more runtime, depending on the number of new agents.

However, what can be said about the solution quality? Since both RS and RSG do not allow changing the plans of the other agents, then using them may lead to solutions of poor quality. Next, we propose a solution quality criteria that online MAPF algorithms can aim for in an effort to achieve better overall solution quality.

\section{Snapshot Optimality}

Definition 6 (Snapshot Optimal). A snapshot optimal plan in an online MAPF setting is a plan for all agents to their goal that is optimal in terms of sum of costs assuming no new agent will appear in the future.

There is no guarantee that always returning a snapshot optimal solution will result in an minimal sum of cost solution for the online MAPF problem. In fact, we know from Observation 5 that such minimality guarantee is not possible in online MAPF. Nonetheless, demanding that an online MAPF algorithm will return snapshot optimal solutions may bias it towards an overall low sum of cost in practice. Indeed, we observe this in our experimental results. Thus, we now propose several algorithms that provide such a guarantee.

Replan All The simplest way, conceptually, to return snapshot optimal solutions is to plan optimally for all agents from their current positions whenever new agent appears. We call this algorithm Replan All (RA), and list its (simple) pseudocode in Alg. 3.

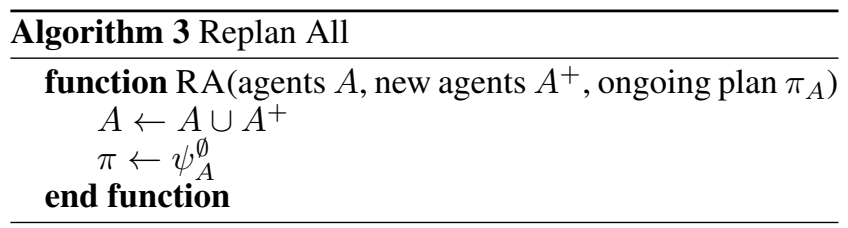

RA is the extreme opposite of RS: it solves a much harder problem - offline MAPF for all current agents without considering the already computed ongoing plan $\left(\pi_{A}\right)$ - but is expected to return a high quality solution, i.e., a plan with a small sum of costs, since it computes the optimal solution for all of the agents currently present and the new agents.

\section{Online Independence Detection}

RA plans optimally for all current agents from their current positions, fully ignoring the plan the existing agents were 
following. This can be wasteful in terms of runtime. Moreover, changing the route of an agent that is already moving, which we refer to as re-routing the agent, may be undesirable, as it requires communication with that agent and modifying the agent's plan may incur some overhead.

Next, we propose an algorithm that returns snapshot optimal solutions but also attempts to minimize the number of re-routes. We call this algorithm Online Independence Detection (OID) for online MAPF, since it is based on Standley's Independence Detection (ID) algorithm (Standley 2010). The main idea of Standley's ID is to plan for each agent separately while ignoring the other agents. If there is a conflict between the generated plans then the conflicting agents are merged into a group and replanned together. This process continues iteratively until there are no conflicts anymore.

The main idea of OID is very similar: allow the new agents to plan while ignoring the other agents. If there is a conflict with the plan of an already planned agent, then we merge the groups of conflicting agents and plan for them altogether (again disregarding the other agents). This is iterated until there are no conflicts anymore.

This adaptation of ID to online MAPF, however, may return plans that are not snapshot optimal. The reason is that an online MAPF algorithm is called multiple times, whenever new agents appear. Consequently, when a new agent $i_{1}$ conflicts with an already existing agent $i_{2}$, it is not sufficient to replan for $i_{1}$ and $i_{2}$ together since $i_{2}$ may have been grouped with other agent, e.g., $i_{3}$ in the previous call. There, perhaps agent $i_{3}$ chose a longer path in the previous call to allow $i_{2}$ use a shorter path. Now, when $i_{2}$ is replanning due to a conflict with the new agent $i_{1}$, perhaps it frees up locations that $i_{3}$ can use to have a shorter path for itself.

To correct this, we modify OID so that it keeps track of the groups used to create the incumbent plan. Algorithm 4 lists the pseudo code for OID. First, the algorithm requires that the set of already planned agents $A$ consists of mutually disjoint and collectively exhaustive sets of agents $g_{1}, g_{2}, \ldots, g_{m}$, and the ongoing plan $\pi_{g_{i}}$ for every $g_{i}$, such that $\pi_{g_{i}}$ is the lowest cost plan for the agents in $g_{i}$. When new agents $A^{+}$ appear, each of them is placed into a new group and an optimal plan for each group is found, disregarding all the other agents (lines 2-6). Then the algorithm iteratively resolves the conflicts until there are no more conflicting groups. Assume there is a conflict of plans between groups $g_{i}$ and $g_{j}$. Then the algorithm tries to find such a plan for $g_{i}$ that avoids conflicts with the agents from group $g_{j}$ while not deteriorating the plan in terms of the sum of costs (line 13). If it does not succeed, it tries analogically to replan for $g_{j}$ while avoiding $g_{i}$ (line 15). If it still did not succeed, it merges the conflicting sets of agents and replans for them together, while, again, disregarding all other agents (lines 17-20). However, this way the algorithm could get stuck in an infinite loop, that is why it is necessary to first check whether the two conflicting groups were already in conflict together before, and thus merge and replan them straight away (lines 9-12).

Theorem 7. OID returns a snapshot optimal solution if it is given a disjoint partition of agents to groups $g_{1}, \ldots g_{m}$ such that for every group $g_{i}$ the cost of its current plan $\pi_{g_{i}}$ is equal to the cost of $\psi_{g_{i}}^{\emptyset}$.

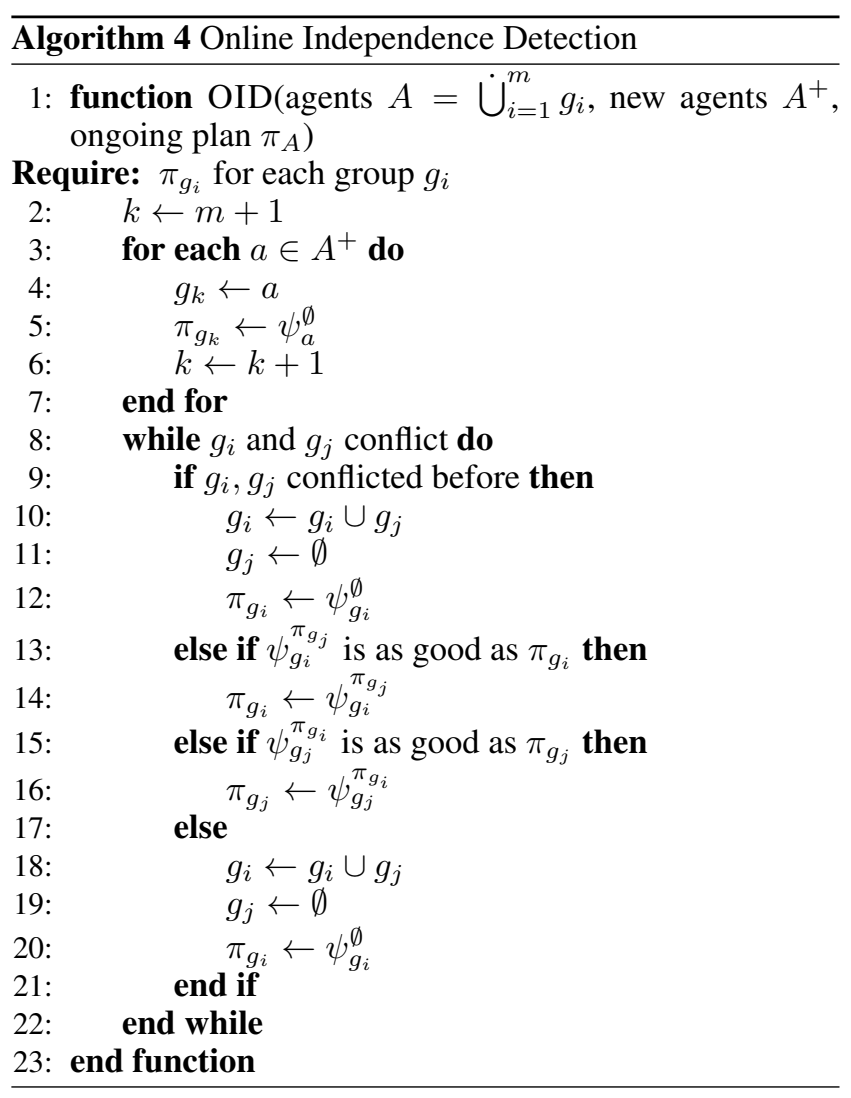

Proof outline. Consider the output of OID after being called by a replan function. OID outputs a new partition of agents to groups $g_{1}^{\prime}, \ldots g_{m^{\prime}}^{\prime}$ that includes the new agents along with a plan to each of these groups. Since OID never breaks a group, then every group in the original set of groups $g_{1}, \ldots g_{m}$ must be equal to or a subset of one of the new groups $g_{1}^{\prime}, \ldots g_{m^{\prime}}^{\prime}$. Since OID returns an optimal plan for each of the new groups, then it cannot miss an optimal plan for any agent.

Corollary 8. If the ongoing plan was snapshot optimal and OID is used to replan when new agents appear then OID is guaranteed to always return snapshot optimal solutions.

Corollary 8 follows by induction due to Theorem 7 and the fact the initial plan is snapshot optimal. Since OID attempts to minimize the number of existing agents it replans for, it has the potential to save runtime and require fewer re-routes than RA.

However, OID has one clear disadvantage compared to RA. If during an online MAPF execution RA is not used to replan for some agents, e.g., due to runtime limitations, RA may still return snapshot optimal solutions if it is used later to replan. By contrast, OID depends on previous calls to replan to return a snapshot optimal solution, as well as a partition of groups and a plan for each group such that each group is solved optimally. If OID does not accept this as input, then it cannot guarantee snapshot optimality. For example, if in one call to replan RS is used instead of OID, the OID loses its snapshot optimality property. 


\section{Suboptimal Independence Detection}

As noted earlier, returning a snapshot optimal plan does not guarantee an optimal solution to the online MAPF problem. Therefore, we propose to change OID by allowing it to return plans whose sum of costs is at most $D$ times more than the optimal sum of costs but allowing it to further reduce the number of agents that have to deviate from the ongoing plan. We call this algorithm Suboptimal OID (SubID).

In details, when OID replans for the group of agents $g_{i}$ while avoiding $g_{j}$ and ignoring all other agents (line 13 and symmetrically line 15), it only accepts plans that have exactly the same sum of costs as that of the optimal plan for $g_{i}$ while ignoring all other agents (the sum of costs value being $f_{1}\left(\psi_{g_{i}}^{\pi_{g_{j}}}\right)$ ). It is likely that such a solution does not exist. SubID allows the new plans to have higher cost, namely any cost in the range $\left[f_{1}\left(\psi_{g_{i}}^{\pi_{g_{j}}}\right), D \cdot f_{1}\left(\psi_{g_{i}}^{\pi_{g_{j}}}\right)\right]$. This is expected to increase the likelihood that such a plan will be found.

Theorem 9. Given a disjoint partition of agents to groups $g_{1}, \ldots g_{m}$ such that for every group $g_{i}$ the cost of its current plan $\pi_{g_{i}}$ is at most $w_{1}$ times the cost of $\psi_{g_{i}}^{\emptyset}$, then SubID with parameter $D=w_{2}$ will return a solution whose cost is at most $w_{1} \cdot w_{2}$.

Proof outline The proof is similar to that of Theorem 7: every group $g_{i}$ is a contained in exactly one group from the solution of SubID. The added suboptimality is a factor of at most $w_{2}$ and the suboptimality of the existing plan is at most $w_{1}$ thus the overall suboptimality is $w_{1} \cdot w_{2}$.

A direct corollary of Theorem 9 is that if SubID is always used to replan when new agents appear then after the $m$ replan calls SubID will output a plan whose cost is at most $D^{m}$ times the cost of a snapshot optimal plan.

\section{Results of Experiments}

We implemented all the algorithms and evaluated their performance on a set of randomly generated problems. All experiments were conducted on Dell PC with an Intel $($ $)$ Core $^{\mathrm{TM}} \mathrm{i} 7-2600 \mathrm{~K}$ processor running at $3.40 \mathrm{GHz}$ with 8 GB of RAM.

\section{Instances}

We created two datasets of online MAPF problems based on 4-connected grids designed to simulate intersections for autonomous vehicles. The first dataset is on small and dense grids, chosen from the 4 types of small grids depicted in Figure 3. Each problem started with no agents present at the outset. Then we are adding new agents, the total number of which is in the range $\{10,12,15,17,20,22,25\}$. The starting time of each agent is set uniformly at random from the interval $[1,30]$, so a number of agents may appear at the same time. The start location is a random location from a randomly picked margin of the map and the goal is randomly picked from the opposite margin. We generated 5 problems for each configuration. Altogether this dataset contained 140 problems.

The second dataset is on a larger grid depicted in Figure 4. The starting time of each agent is set uniformly at random from the interval $[1,100]$. The number of new agents

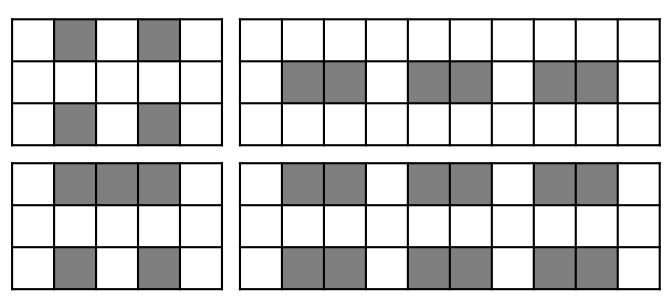

Figure 3: Small grids.

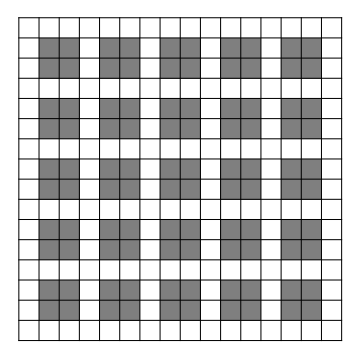

Figure 4: Large grids.

to appear over these timesteps increments from 60 to 70 . The agents are moving from a randomly picked margin to another randomly picked margin. No agent is present at the outset. We generated 5 problems for each configuration. Altogether we generated 30 testing instances.

\section{Implementation Details}

All the proposed algorithms require an optimal offline MAPF solver, adapted to our assumptions that a new agent appears in its garage and agents disappear at their goals. We created two such modified optimal offline MAPF solvers: one based on a reduction to Boolean satisfiability (SAT) via the Picat language and compiler (version 2.2\#3) (Barták et al. 2017), and the other based on the Conflict-Based Search (CBS) algorithm (Sharon et al. 2015). For the first dataset (small grids) we used the Picat-based solver and for the second dataset (large grid) we used the CBS-based solver. This is because Picat-based solvers perform better on small and dense grids and CBS-based solver performs better on large grids (Barták et al. 2017).

\section{Dataset \#1: Small Grids}

We ran the following algorithms on the small grids dataset: Replan Single (RS), Replan Single Grouped (RSG), Replan All (RA), Online Independence Detection (OID), and Suboptimal ID (SubID) with $D$ chosen to be 1.1 . For every instance, we always run first RS and then the algorithm in question. Whenever the runtime for a newly appeared agent exceeds the given time limit, which was set to 30 seconds, the output from RS is taken into results. The reason is that $\mathrm{RS}$ is done extremely quickly and it is always good to have some solution rather than no solution. The number of instances, where the timelimit was reached and thus the result from RS was taken, can be seen in Table 2 .

How the algorithms behave with respect to the number of times an agent had to change its plan (referred to hereafter 


\begin{tabular}{l|rrrr} 
\#agents & RSG & SubID & OID & RA \\
\hline 10 & 0.00 & 1.70 & 1.70 & 0.55 \\
12 & 0.00 & 2.55 & 2.20 & 1.05 \\
15 & 0.05 & 5.90 & 4.25 & 2.40 \\
17 & 0.35 & 6.55 & 7.10 & 3.40 \\
20 & 1.20 & 9.55 & 9.80 & 5.65 \\
22 & 1.70 & 10.85 & 10.55 & 9.40 \\
25 & 3.50 & 11.65 & 11.65 & 10.00
\end{tabular}

Table 2: The average number of timeouts per instance of each algorithm.

as the number of changes) is shown in Table 3 . The number of changes for RS and RSG is always zero, since it never replans for the other agents, so we do not report it in Table 3. The results clearly show that OID requires fewer changes than RA, and SubID requires even fewer changes than OID. For example, with 15 agents, SubID required on average 1.5 changes, while OID required 3.45 and RA 6.45. The slight decrease in the number of changes for 20 agents is caused by the increase in the number of timeouts and therefore more RS calculations that avoid changes is used.

The relative gain in terms of the sum of costs over RS is shown in Table 4. The expectations that RA will always be the best w.r.t. the sum of costs have been confirmed. It is worth noticing that SubID, which does not return an optimal solution in every replan, brings more or less the same gains in terms of the sum of costs as OID. Thus, SubID shows promising results, with comparable sum of costs to OID but significantly fewer changes.

\begin{tabular}{l|rrr} 
\#agents & SubID & OID & RA \\
\hline 10 & 2.65 & 3.30 & 5.15 \\
12 & 2.05 & 2.80 & 3.80 \\
15 & 1.50 & 3.45 & 6.45 \\
17 & 3.80 & 3.85 & 10.05 \\
20 & 2.85 & 2.65 & 10.15 \\
22 & 2.95 & 4.25 & 6.80 \\
25 & 1.90 & 2.55 & 6.40
\end{tabular}

Table 3: Avg. \# of re-routes for smaller grids using Picat.

\begin{tabular}{l|rrrr} 
\#agents & RSG & SubID & OID & RA \\
\hline 10 & 1.01 & 1.06 & 1.06 & 1.10 \\
12 & 1.02 & 1.08 & 1.09 & 1.14 \\
15 & 1.03 & 1.04 & 1.11 & 1.17 \\
17 & 1.05 & 1.14 & 1.13 & 1.22 \\
20 & 1.04 & 1.04 & 1.04 & 1.19 \\
22 & 1.06 & 1.06 & 1.10 & 1.15 \\
25 & 1.05 & 1.07 & 1.08 & 1.15
\end{tabular}

Table 4: Avg. gain in SOC over RS for smaller grids using Picat.

\section{Dataset \#2: Large Grids}

The experiments on large grids dataset were carried out in the same way. The number of instances where time limit

\begin{tabular}{l|rrrr} 
\#agents & RSG & SubID & OID & RA \\
\hline 60 & 0.0 & 1.0 & 0.4 & 0.8 \\
62 & 0.0 & 0.0 & 0.2 & 0.6 \\
64 & 0.0 & 1.0 & 0.8 & 0.4 \\
66 & 0.0 & 0.0 & 0.2 & 0.6 \\
68 & 0.0 & 0.0 & 0.0 & 0.4 \\
70 & 0.0 & 0.6 & 1.0 & 0.4
\end{tabular}

Table 5: Avg. \# of timeouts on the larger grid using CBS.

\begin{tabular}{l|rrr} 
\#agents & SubID & OID & RA \\
\hline 60 & 5.6 & 13.4 & 30.0 \\
62 & 4.6 & 11.4 & 26.0 \\
64 & 4.4 & 11.6 & 25.0 \\
66 & 5.0 & 16.6 & 32.0 \\
68 & 6.4 & 12.4 & 27.4 \\
70 & 5.8 & 12.0 & 24.6
\end{tabular}

Table 6: Avg. number of re-routes for larger grid using CBS.

was reached is shown in Table 5. The number of changes is shown in Table 6, and the relative gain w.r.t. the sum of costs is shown in Table 7. Briefly speaking, the results are in concordance with those of small grids.

While here again we see a clear advantage in terms of the sum of costs for RA, OID, and SubID compared to RS, the differences are significantly smaller. This is because larger grids are sparser, and thus it is easier for RS to find a higher quality solution. The differences in the sum of costs between RA, OID, and SubID are negligible, but SubID still shows a significant advantage in terms of the number of changes. For example, with 66 agents SubID required on average only 5 changes while RA required 32 , and their sum of costs was virtually the same.

In conclusion, SubID confirms to have brought the best trade-off between the quality of the resulting plans and the computational efficiency.

\section{Related Work}

The term online planning in general refers to planning that is done while executing a plan, in contrast to offline planning where all the planning is done upfront. Many papers on online planning defer planning to execution to minimize the computational effort of creating a complete plan for every contingency offline. This includes the seminal work of Korf on Real-Time A* and its many successors (Korf 1990). This is different from our setting, where some of the planning is

\begin{tabular}{l|rrrr} 
\#agents & RSG & SubID & OID & RA \\
\hline 60 & 0.997 & 1.008 & 1.015 & 1.015 \\
62 & 0.994 & 1.008 & 1.012 & 1.012 \\
64 & 1.000 & 1.009 & 1.012 & 1.013 \\
66 & 1.000 & 1.008 & 1.009 & 1.009 \\
68 & 0.996 & 1.007 & 1.009 & 1.009 \\
70 & 0.998 & 1.009 & 1.010 & 1.011
\end{tabular}

Table 7: Avg. gain in SOC over RS on larger grid using CBS. 
done online because the agents do not know in advance how many and where the new agents will appear.

Online MAPF can be viewed as an instance of ad-hoc teamwork (Stone et al. 2010), which exactly addresses cases where the agents constantly need to coordinate with new agents. However, the form of interactions between the agents in online MAPF is very specific - they cannot collide with each other, while ad-hoc teamwork usually involves deeper forms of collaboration. In the motion planning literature, there is work on single robot online planning that replans for newly observed obstacles by using pre-defined planning patterns (Majumdar and Tedrake 2013). In addition, some prior work on multi-robot motion planning that are fundamentally forms of prioritized planning (Van Den Berg and Overmars 2005) can be adapted to the online case, resulting in a behavior similar to RS. Other motion planning techniques (Dobson et al. 2017; Godoy et al. 2015) are designed for offline, continuous spaces and adapting them to a discrete and online MAPF is non-trivial.

\section{Conclusion and Future Work}

In this paper, we study the online version of the multi-agent pathfinding problem, in which new agents appear during execution of a plan. We analyzed several variants of the problem theoretically, showing which of these variants can be solved by an online MAPF algorithm. Then, we proposed several online MAPF algorithms. The pros and cons of each algorithm are analyzed and demonstrated experimentally. While the baseline replan single algorithm is the fastest, its solution quality is, in some configurations, poor compared to the replan all baseline. OID and SubID provide further improvements to replan all, as they minimize the sum of costs while also aiming to force fewer agents to change their planned paths. Future work we expand on this is modifying the replanning process to try to find individual plans that are close to the ongoing plan (Felner et al. 2007) and/or plans that minimize the probability of conflicts in the future.

\section{Acknowledgement}

This research is supported by the Czech-Israeli Cooperative Scientific Research Project 8G15027, by SVV project number 260 453, by the EU and the Ministry of Industry and Trade of the Czech Republic under the Project OP PIK CZ.01.1.02/0.0/0.0/15 019/0004688, and by ISF grant no. $210 / 17$ to Roni Stern. Roman Barták is supported by the Czech Science Foundation project 18-07252S.

\section{References}

Barták, R.; Zhou, N.-F.; Stern, R.; Boyarski, E.; and Surynek, P. 2017. Modeling and solving the multi-agent pathfinding problem in picat. In International Conference on Tools with Artificial Intelligence (ICTAI), 959-966.

Dobson, A.; Solovey, K.; Shome, R.; Halperin, D.; and Bekris, K. E. 2017. Scalable asymptotically-optimal multirobot motion planning. In International Symposium on MultiRobot and Multi-Agent Systems (MRS), 120-127. IEEE.
Dresner, K. M., and Stone, P. 2008. A multiagent approach to autonomous intersection management. J. Artif. Intell. Res. 31:591-656.

Erdem, E.; Kisa, D. G.; Öztok, U.; and Schüller, P. 2013. A general formal framework for pathfinding problems with multiple agents. In $A A A I$.

Felner, A.; Stern, R.; Rosenschein, J. S.; and Pomeransky, A. 2007. Searching for close alternative plans. Autonomous Agents and Multi-Agent Systems 14(3):211-237.

Felner, A.; Stern, R.; Shimony, S. E.; Boyarski, E.; Goldenberg, M.; Sharon, G.; Sturtevant, N. R.; Wagner, G.; and Surynek, P. 2017. Search-based optimal solvers for the multiagent pathfinding problem: Summary and challenges. In International Symposium on Combinatorial Search (SoCS), 2937.

Godoy, J. E.; Karamouzas, I.; Guy, S. J.; and Gini, M. 2015. Adaptive learning for multi-agent navigation. In the International Conference on Autonomous Agents and Multiagent Systems (AAMAS), 1577-1585.

Korf, R. E. 1990. Real-time heuristic search. Artif. Intell. 42(2-3):189-211.

Ma, H.; Li, J.; Kumar, T.; and Koenig, S. 2017. Lifelong multi-agent path finding for online pickup and delivery tasks. In Proceedings of the 16th Conference on Autonomous Agents and MultiAgent Systems, 837-845. International Foundation for Autonomous Agents and Multiagent Systems.

Majumdar, A., and Tedrake, R. 2013. Robust online motion planning with regions of finite time invariance. In Algorithmic Foundations of Robotics X. Springer. 543-558.

Pallottino, L.; Scordio, V. G.; Bicchi, A.; and Frazzoli, E. 2007. Decentralized cooperative policy for conflict resolution in multivehicle systems. IEEE Transactions on Robotics 23(6):1170-1183.

Sharon, G.; Stern, R.; Felner, A.; and Sturtevant, N. R. 2015. Conflict-based search for optimal multi-agent pathfinding. Artificial Intelligence 219:40-66.

Silver, D. 2005. Cooperative pathfinding. In Artificial Intelligence and Interactive Digital Entertainment (AIIDE), 117122.

Standley, T. S. 2010. Finding optimal solutions to cooperative pathfinding problems. In AAAI Conference on Artificial Intelligence.

Stone, P.; Kaminka, G. A.; Kraus, S.; Rosenschein, J. S.; et al. 2010. Ad hoc autonomous agent teams: Collaboration without pre-coordination. In $A A A I$.

Surynek, P.; Felner, A.; Stern, R.; and Boyarski, E. 2016. Efficient SAT approach to multi-agent path finding under the sum of costs objective. In the European Conference on Artificial Intelligence (ECAI), 810-818.

Van Den Berg, J. P., and Overmars, M. H. 2005. Prioritized motion planning for multiple robots. In IEEE/RSJ International Conference on Intelligent Robots and Systems (IROS), 430-435.

Yu, J., and LaValle, S. M. 2012. Multi-agent path planning and network flow. In Workshop on the Algorithmic Foundations of Robotics (WAFR), 157-173. 